\title{
Repensando las evaluaciones finales en el ámbito de las ingenierías. El caso en la Universidad Católica de Salta
}

\author{
Rethinking final evaluations in the engineering field. The case in the \\ Catholic University of Salta
}

Roberto Daniel Breslin ${ }^{1}$ y Mariela Elsa Capaldo ${ }^{2}$

Citar: Breslin, R. D. y Capaldo, M. E. (2020). Repensando las evaluaciones finales en el ámbito de las ingenierías. El caso en la Universidad Católica de Salta. Cuadernos Universitarios, 13, pp. 89-98.

Recibido: setiembre 2020

Aceptado: octubre 2020

Ensayo científico

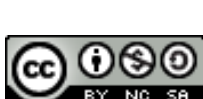

\section{Resumen}

Con el fin de repensar las prácticas evaluativas de los docentes de una carrera de Ingeniería de la Universidad Católica de Salta (UCASAL), a mediados del mes de julio de 2020 se realizó un taller de evaluación. Luego de realizadas las actividades propuestas y de transcurrido el siguiente turno de exámenes, se encuestó a los docentes involucrados. Este documento presenta el análisis de las respuestas de los docentes a la encuesta y sus prácticas concretas durante una instancia de evaluación final. Las consideraciones giran en torno a los instrumentos y las metodologías de

\footnotetext{
${ }^{1}$ Facultad de Ingeniería, Universidad Católica de Salta (UCASAL), Argentina. Ingeniero Electricista con Orientación en Electrónica por la Universidad Nacional de Tucumán. Master en Dirección Estratégica en Telecomunicaciones por Grupo IOE (Iniciativas Organizativas de Empresa) y Universidad Americana de Europa (UNADE). Especialista Docente de Nivel Superior en Educación y TICs por Ministerio de Educación Ciencia y Tecnología. Investigador del Instituto de Estudios Interdisciplinarios de Ingeniería (I.Es.I.Ing.) de la UCASAL. Docente Adjunto de Sistemas de Conmutación y Docente Auxiliar de Antenas en la Carrera Ingeniería en Telecomunicaciones de UCASAL. Jefe de la Carrera de Ingeniería en Telecomunicaciones de UCASAL. rbreslin@ucasal.edu.ar

${ }^{2}$ Escuela de Educación y Perfeccionamiento Docente, Universidad Católica de Salta (UCASAL), Argentina.

Profesora en Ciencias de la Educación por la Universidad Nacional de Salta. Magister en Nuevas tecnologías aplicadas a la educación por el IUP (Instituto Universitario de Postgrado: Universidad de Alicante, Universidad Autónoma de Barcelona y Universidad Carlos III de Madrid). Investigadora del Consejo de Investigaciones de la UCASAL. Docente a cargo de Metodología de la Investigación y Seminario de Tesis en la Licenciatura en Gestión Educativa. Directora de la Escuela de Educación y Perfeccionamiento Docente de la UCASAL. mecapaldo@ucasal.edu.ar Usuario ORCID: marielacapaldo
} 
evaluación utilizadas, las competencias profesionales y el propósito de la evaluación final.

Palabras clave: prácticas de evaluación - evaluación de los aprendizajes - evaluación en la universidad - evaluaciones en ingeniería

\section{Abstract}

In order to rethink the evaluative practices of the Catholic University of Salta (UCASAL) engineering professors, an evaluation workshop was held in midJuly 2020. After carrying out the proposed activities and after the next examination session, the teachers involved were surveyed. This document presents the analysis of the teachers' answers to the survey and their concrete practices during a final evaluation instance. The considerations revolve around the instruments and evaluation methodologies used, the professional competencies and the purpose of the final evaluation.

Key words: evaluation practices - assessment of learning - evaluation at the university - evaluations in engineering

\section{Introducción}

La educación universitaria ha iniciado hace tiempo procesos de revisión de sus prácticas educativas, donde la evaluación ha sido el detonante de importantes contradicciones vinculadas con otros aspectos del proceso formativo.

El campo de las ingenierías no es ajeno a esta situación, y se suma a ello la irrupción de modalidades evaluativas mediadas por la tecnología, que sin lugar a dudas se presentan con ventajas cualitativas relevantes para el proceso formativo de los ingenieros. Pero, por otro lado, también nos encontramos con rechazo y desconfianza que se generan en ciertos grupos de docentes, siendo algunos de ellos responsables de materias que incluyen contenidos y herramientas tecnológicas del campo de la informática y las telecomunicaciones. Por ejemplo, los avances en las tecnologías de la información y la comunicación son retomadas, utilizadas, valoradas y puestas en juego cuando se trata de la enseñanza, del trabajo, del hacer y experimentar con ellas en los procesos formativos de los estudiantes; pero son sometidas a duros juicios de valor negativos cuando se trata de su uso en procesos evaluativos.
En este actual contexto de pandemia, la UCASAL mudó todo el sistema de educación presencial a su plataforma virtual, y los procesos de enseñanza fueron garantizados para todas las carreras. En este sentido, las instancias de exámenes finales también fueron transformadas para sostener esta lógica, en algunos turnos los exámenes finales fueron $100 \%$ virtuales y en otros turnos se alternó el sistema presencial con el virtual, para garantizar a todos los estudiantes el derecho de rendir finales, así como la conformación de tribunales evaluadores con los docentes correspondientes.

Las evaluaciones de los aprendizajes mediadas por el uso de la tecnología pasaron al centro del análisis, del debate, y en algunos casos al centro de la hoguera. Resultó evidente que nos encontramos con dos sectores, los docentes a favor y los docentes en contra; estos últimos rechazan de plano este tipo de modalidad en la evaluación. Poder identificar algunos supuestos que caracterizan a estos sectores es el objetivo de este estudio, realizado en una carrera en particular de la Facultad de Ingeniería.

El avance hacia procesos formativos mediados por las tecnologías es una realidad que llegó hoy a las universidades, a las carreras, 
espacios curriculares y sectores que estaban seguros en la modalidad presencial. Ya no se trata solamente de ajustar la formación a las demandas del mundo del trabajo, a las particularidades de los nuevos jóvenes estudiantes, a la era digital o a la sociedad del conocimiento; se trata de redefinir las instituciones educativas desde sus fundamentos medulares. Somos los docentes, con nuestras prácticas de enseñanza y de evaluación cotidiana, quienes recreamos las respuestas a esta educación en y post pandemia.

En este contexto, otro aspecto que impacta fuertemente en las ingenierías está relacionado con las competencias específicas y genéricas que se establecieron como estándares de acreditación de las carreras (CONFEDI, 2018). Las entidades receptoras de los profesionales, generadoras de fuentes laborales, tienen la responsabilidad social y legal por muchas de las actividades involucradas en esta profesión, denominadas actividades reservadas. Varias de ellas son catalogadas de interés público, y es en este sentido que el sistema educativo debe garantizar el desarrollo adecuado y eficaz de esas actividades reservadas desde el desarrollo de competencias profesionales de las ingenierías. Estas competencias están explícitamente planteadas en los planes de estudio y deben ser fuertemente desarrolladas en los diferentes espacios curriculares y, en este sentido, deben ser claramente evaluadas (Giordano, 2016).

E1 desempeño profesional de estas actividades reservadas no solo está regulado por normativas específicas a nivel nacional, sino que es la propia sociedad la que deposita en el sistema educativo (las universidades) la potestad de asegurar los conocimientos y las competencias efectivamente logradas por los profesionales (Ministerio de Educación de la Nación Argentina, 2006).

\section{E1 taller de evaluación}

Con el fin de repensar nuestras prácticas evaluativas, y ante la necesidad de ajustarlas a las nuevas características y condiciones de trabajo, como la virtualidad, se decidió realizar un encuentro de taller con docentes de la carrera durante el mes de julio de 2020. El primer elemento a considerar tuvo que ver con la modalidad de las evaluaciones que efectivamente se desarrollan, y que apuntan a considerar solamente conocimientos memorísticos por parte de los estudiantes y a generar un clima de estrés alto.

Los conceptos de memoria y de estrés en los exámenes han sido desde hace tiempo objeto de estudios; como afirman García Ramos y Zurita Aimacaña (2020), existe una correspondencia significativamente negativa entre los valores utilizados en el estudio que confirman la hipótesis de investigación acerca de la existencia de correlación entre la memoria de trabajo y el estrés académico.

Las ciencias cognitivas han denominado memoria de trabajo (MT), o working memory (WM), al mantenimiento activo y al procesamiento ejecutivo de la información disponible en el sistema cognitivo. Una extensa literatura muestra que la MT es un constructo muy importante en diversas áreas, $\mathrm{y}$ varios estudios han demostrado su rol clave en un amplio rango de procesos cognitivos, tales como la comprensión, el razonamiento y la resolución de problemas (Engle, 2002). La MT es una variable predictiva importante en la habilidad intelectual y del desempeño académico, consistente a través del tiempo (Adams y Hitch, 1997), lo que lleva a interpretar que esta memoria está directamente relacionada con las competencias específicas que se deben adquirir en la formación de un ingeniero. 
Tal como afirman Musso, Costa Lieste y Duarte (2012) en un estudio realizado en la Argentina, «las principales dificultades de los estudiantes respecto a sus estrategias de aprendizaje se encuentran en la selección y expresión de ideas principales y en las estrategias de manejo del tiempo y de la ansiedad frente a los exámenes» (p. 7). Las dificultades en la selección y expresión de ideas principales implican problemas a la hora de comprender el texto, y son estrategias que demandan procesos cognitivos de memoria de trabajo y focalización de la atención sobre lo más relevante del texto. En tanto, las dificultades en el manejo del tiempo estarían planteando problemas a la hora de planificar y organizar secuencias de metas, factibles de ser logradas en función del tiempo disponible. En este sentido podemos entender por qué una instancia de examen constituye una situación altamente «traumática», en aspectos fundamentales como el manejo del tiempo y la memoria de trabajo.

Por otra parte, el trabajo de Palacios Navarro (2002), vinculado a los efectos de la evaluación formativa virtual en el rendimiento académico, expresa que desde una perspectiva psicodidáctica las nuevas tecnologías siguen ofreciendo la posibilidad de aproximarse al ideal constructivista del proceso de enseñanza y aprendizaje, entendiéndolo como un proceso de construcción y atribución de significados. Claro que esta situación no está exenta de dificultades, dado que al momento de plantear exámenes basados en nuevas tecnologías se requiere de logística y conocimientos particulares de los docentes universitarios, además de consumir un tiempo extra de trabajo y planificación, que no siempre está suficientemente reconocido.

Sobre la base de los elementos desarrollados hasta aquí, desde la jefatura de carrera de una ingeniería, se convocó a la participación de un taller de evaluación, con el objetivo primordial de repensar los modelos de evaluación y las modalidades que se utilizan. Todo esto en el contexto de sostener evaluaciones no presenciales, avanzar hacia la evaluación de las competencias profesionales y con intenciones de bajar los niveles de ansiedad de estudiantes y docentes, quienes ponen sobre el tapete las dudas en relación con las evaluaciones virtuales. Participaron 46 docentes de los 71 convocados de la carrera, lo que equivale al $65 \%$ de asistencia, y 42 de ellos eran jefes de cátedra.

La tarea central del taller consistió en repensar al menos dos ítems o preguntas concretas de su instrumento de evaluación, para que pudieran apuntar a evaluar algunas de las competencias profesionales presentadas y que no dieran importancia a la posibilidad de consulta externa por parte de los estudiantes. En este punto conviene realizar un paréntesis explicativo, dado que constituye una de las más fuertes dudas y cuestionamientos que se realizan desde los equipos docentes a las evaluaciones mediadas por tecnologías.

La posibilidad de consultas externas de los estudiantes - a textos, bibliografías, sitios web, apuntes de cátedras, compañeros, etc.durante el momento del examen se presenta como uno de los principales cuestionamientos, por parte de los docentes, a las evaluaciones virtuales. En este sentido, desde la jefatura de carrera, en el marco del taller, se desestimó este argumento cuando se propuso evaluar competencias profesionales, como por ejemplo la resolución de problemas o el trabajo en equipo, cuestiones centrales en la formación de un ingeniero. No resulta relevante si el estudiante consulta un sitio web o un software específico para dar una respuesta, dado que esta no refiere a un dato o contenido lineal y 
memorístico, sino a la puesta en juego de un «saber hacer» con ese dato o conocimiento.

\section{Las respuestas del equipo docente}

Posteriormente a la realización del taller, se concretó una encuesta a todos los docentes de una carrera de Ingeniería. De los 35 que respondieron la encuesta, el $94 \%$ había participado en el taller de evaluación del mes de julio. Es necesario aclarar que además de la asistencia se planteó la realización de una actividad vinculada con revisar, analizar y modificar algunas consignas de un instrumento de evaluación y la metodología empleada en los exámenes finales.

Dado que dentro de los objetivos del taller se encontraba la posibilidad de repensar los modos e instrumentos de evaluación en relación con focalizar las competencias profesionales, resultó necesario consultar acerca del nivel de conocimientos de estas por parte de los docentes. Resulta interesante observar que el $29 \%$ de los docentes no conocía las competencias profesionales específicas de ingeniería, en tanto que el $71 \%$ restante manifestó que ya las conocía o que se había actualizado en el encuentro. En relación con las competencias genéricas vinculadas con la formación universitaria, solo el $23 \%$ de los docentes no las conocía, y el resto $-77 \%$ del plantel docente-, sí. Es necesario puntualizar la importancia de esta situación dado que, como fue señalado anteriormente, una actividad reservada como las ingenierías debe contar con formadores que conozcan al $100 \%$ las particularidades del perfil profesional.

La tarea concreta que se debía realizar luego del taller de evaluación consistía en repensar y reelaborar algunas de las consignas del instrumento de evaluación final de la materia en la cual se desempeñaba cada uno como docente, reelaboración que debía estar enmarcada en el planteo de competencias profesionales.

El $68 \%$ de los docentes que respondieron la encuesta realizaron efectivamente la reelaboración de una, dos o más preguntas de sus exámenes. Este porcentaje pone de manifiesto el interés por revisar las prácticas evaluativas que se llevan a cabo en la carrera. En tanto que el $23 \%$ manifestó que las consignas de sus instrumentos de evaluación ya estaban orientadas a las competencias, desde antes del taller de evaluación. E1 $9 \%$ restante manifestó que no había tenido tiempo suficiente para concretar la tarea, o que las características de su materia no permitían incorporar las competencias profesionales específicas de la ingeniería. En este punto nos encontramos con espacios curriculares comunes a las diversas ingenierías que se dictan en la unidad académica, en los cuales no siempre se retoman las competencias específicas, pero sí debieran incluirse las competencias genéricas definidas en la normativa vigente.

En este punto es importante preguntarse, entonces, ¿por qué si más del $70 \%$ de estos docentes ya conoce las competencias específicas de ingeniería, solo un $23 \%$ las toma en consideración para elaborar sus instrumentos de evaluación? Puede pensarse que esto tiene que ver con la histórica grieta que separa la enseñanza de la evaluación, o que tiene que ver con las concepciones de evaluación que sostienen los docentes, o con el bajo grado de centralidad que se otorga a la evaluación en relación con otras actividades de la práctica docente. Estas cuestiones presentan temas para el constante análisis y evaluación de las prácticas docentes.

La actividad propuesta en el taller también tenía como objetivo lograr algunos cambios en los exámenes finales que se desarrollarían en el cuarto turno ordinario, ya sea en relación con la metodología o en relación con los ins- 
trumentos. Esto se vincula directamente con la necesidad de instalar momentos y espacios para el análisis de las prácticas docentes, de manera sistemática y regular, como un accionar constitutivo de la vida universitaria. Una de las preguntas de la encuesta fue pensada para recabar información al respecto, solicitando explicitar lo que había ocurrido en el citado turno. En este sentido, el $54 \%$ de los docentes manifestó que había cambiado preguntas o consignas de sus instrumentos de evaluación y, el $11 \%$, que había cambiado la metodología de evaluación y las consignas, en tanto que solo el $6 \%$ había cambiado la metodología sin modificar los instrumentos. Más allá del análisis pormenorizado que queda por realizar, lo importante es mencionar que el $71 \%$ de los docentes que respondieron la encuesta realizaron alguna modificación en sus prácticas evaluativas en el turno de exámenes finales con posterioridad a la realización del taller. Esta característica de proactividad en los docentes es fundamental para avanzar en la mejora de las prácticas y en la investigación formativa que se pretende llevar adelante (González Agudelo; 2006).

Resulta interesante someter las respuestas obtenidas a un análisis de coherencia entre las vinculadas a la actividad de repensar las consignas de un instrumento de evaluación (ARC) y las vinculadas a la modificación real de instrumentos o modalidades durante el cuarto turno de exámenes (M4T). Para ello se utilizó el criterio de coherencia segmentado en:

- Coherencia positiva: sí realizaron ARC y también realizaron M4T - 54\%

- Coherencia negativa: no realizaron ARC y no realizaron M4T - $20 \%$

- Incoherencia positiva: no realizaron ARC y sí realizan M4T - $11 \%$

- Incoherencia negativa: sí realizaron ARC y no realizan $\mathrm{M} 4 \mathrm{~T}-14 \%$
Volveremos sobre estos porcentajes un poco más adelante, con otro análisis de coherencia vinculado con este.

La modalidad del examen y el formato elegido por los docentes son, sin lugar a dudas, los elementos más controversiales de esta situación. Tal como fue planteado más arriba, la virtualidad genera desconfianza en el cuerpo docente. Entre aquellos que manifestaron haber cambiado la metodología de examen en el cuarto turno, el $83 \%$ de los encuestados, se observa que las herramientas tecnológicas más utilizadas fueron:

- $1^{\circ}$ Videoconferencia y cuestionario on line; cuestionario Moodle, $31 \%$

- $2^{\circ}$ Videoconferencia y tarea a entregar (por cualquier medio), $28 \%$

- $3^{\circ}$ Videoconferencia únicamente; videoconferencia y cuestionario en pantalla; cuestionario en otra plataforma (fuera de la institucional), $11 \%$

- $4^{\circ}$ Tarea a entregar por cualquier medio, $9 \%$ La elección de una herramienta que permite recrear la presencialidad con un encuentro sincrónico mediado por dispositivos tecnológicos (la videoconferencia) es un aspecto que pone de manifiesto, una vez más, la desconfianza que pesa en la virtualidad al momento de una evaluación de aprendizajes. Asimismo, el uso de la videoconferencia asociada a un cuestionario o a una tarea adicional intentan simular un examen presencial, en el cual el docente entrega un cuestionario o consignas de trabajo en formato papel, el estudiante las desarrolla y las devuelve al docente para su revisión. Más del $70 \%$ de los docentes, habiendo modificado las metodologías de evaluación en el cuarto turno de exámenes, sostienen un formato que recrea la presencialidad.

Al realizar el mismo análisis de coherencia entre las respuestas vinculadas con repensar consignas de su instrumento de evaluación 
(ARC) y las respuestas vinculadas a la elección de las herramientas tecnológicas señaladas en el párrafo anterior, se encuentran los siguientes porcentajes:

- Coherencia positiva: sí realizaron ARC y cambiaron metodología, $43 \%$

- Coherencia negativa: no realizaron ARC y no cambiaron metodología, $26 \%$

- Incoherencia positiva: no realizaron ARC y sí cambiaron metodología, $3 \%$

- Incoherencia negativa: sí realizaron ARC y no cambiaron metodología, $29 \%$

En este punto es cuando se vuelve al primer análisis de coherencia, y se puede evidenciar la disminución de la coherencia positiva y el incremento de la incoherencia negativa. Lo que efectivamente ocurre en una instancia real de evaluación final, en un examen concreto, no se vincula directamente con la tarea realizada para el taller de evaluación en relación con repensar las prácticas evaluativas. La negatividad aumenta en el orden del $20 \%$ en este último análisis.

Cuando se consulta el nivel de satisfacción o insatisfacción de lo acontecido en el cuarto turno de exámenes finales a aquellos docentes que sí cambiaron la metodología de evaluación, nos encontramos con las siguientes respuestas:

- Está satisfecho con el cambio, el $40 \%$

- Está insatisfecho con el cambio, el $14 \%$

- Tiene una impresión mixta de satisfacción e insatisfacción, el $20 \%$

- Manifiesta no haber realizado cambios en la metodología de evaluación, el $26 \%$. En este punto aparece un dato contrario al recogido anteriormente.

La satisfacción está dada por elementos tales como la facilidad en el desarrollo del examen, la corrección automática del instrumento, el presentar un diseño más amigable para el estudiante, la sensación de tranquilidad durante el examen. En tanto, la insatisfacción está dada por elementos como los resultados en las notas, los resultados en relación con los conocimientos evaluados, las dudas respecto al desempeño de los estudiantes y el tiempo de desarrollo del examen.

Cabe poner atención en el elevado grado de satisfacción de los docentes en relación con las herramientas utilizadas, del $40 \%$ concretamente, considerando que las descritas anteriormente constituyen una simulación de la presencialidad. En este sentido se puede avanzar en preguntarnos si la satisfacción está dada por esa simulación o por las posibilidades reales del uso de la virtualidad para evaluar.

Entre los docentes que no cambiaron su metodología de evaluación, se pueden identificar los siguientes motivos:

- Considero necesario ver el rostro del estudiante en un examen, $34 \%$

- Tengo la impresión que de otro modo el estudiante puede tomar algún tipo de atajo, $9 \%$

- La tarea es la única forma de evaluar mi materia, $0 \%$

- No estoy seguro de estar evaluando correctamente, $0 \%$

- No estoy seguro de estar evaluando en forma integral, $0 \%$

Parece que el hecho de haber cambiado la metodología o no en el turno de examen analizado no interfiere con la alta valoración y el uso que se da a la videoconferencia, al «mirar» a los estudiantes en tiempo real.

Hacia el final de la encuesta se encuentran dos preguntas abiertas. La primera está orientada a identificar los objetivos de una instancia de evaluación final, en tanto que la segunda se vincula con poner de manifiesto las metodologías más adecuadas para este tipo de evaluación.

Cuando centramos la mirada en el momento en el cual se llevan a cabo las evaluaciones, 
nos encontramos con tres opciones: evaluación inicial, evaluación procesual y evaluación final. Cada una de ellas tiene objetivos específicos, tanto para el proceso de enseñanza como para el proceso de aprendizaje que lleva adelante el estudiante. Esta tipología no es excluyente de la evaluación formativa y sumativa, dado que desde cualquiera de estos posicionamientos vamos a encontrar evaluaciones que se llevan a cabo antes, durante o al finalizar un proceso, tarea o proyecto, entre otros.

En este sentido encontramos que solamente el $29 \%$ de las respuestas se vinculan directamente con los propósitos de una evaluación final, con expresiones tales como «verificar lo aprendido», «acreditar algunas competencias», "comprobar si lo enseñado fue adquirido por el alumno». En tanto, el $31 \%$ de los docentes sostiene que el objetivo del examen final es «evaluar»: evaluar conocimientos, procedimientos, razonamiento, competencias, conceptos, lo aprendido por los alumnos. El término «evaluar» es tan multifacético como teorías y corrientes pedagógicas conocemos, por lo cual no es posible otorgarle un significado unívoco para cada uno de los encuestados.

Resulta muy significativo que el $40 \%$ de las respuestas obtenidas no identifiquen una finalidad concreta para el examen final, sino que hagan alusión a objetivos de aprendizaje de los estudiantes. Aparecen expresiones tales como «que el alumno conozca los fundamentos y conceptos básicos...», «que el alumno salga con los conceptos aprehendidos...», «... que el alumno tenga compresión de los pilares de la materia...», "que el estudiante demuestre que estudió...».

La pregunta de para qué se evalúa remite a las finalidades de la evaluación, cuestión que constituye uno de los ejes centrales para poder tomar las decisiones adecuadas. En términos generales podemos identificar algunas finali- dades principales de todo proceso evaluativo: diagnóstico, pronóstico, selección y acreditación. Estas finalidades no necesariamente se plantean como excluyentes, pero sí definen opciones metodológicas diferenciadas (Elola y Toranzos, 2009).

La acreditación es un requisito formal que suele descansar en valoraciones cuantitativas, con escalas de valoración definidas, que tiene como finalidad asegurar que el sujeto alcance determinados objetivos establecidos a priori, en una materia, en una carrera, en una institución. Las instancias de exámenes finales universitarios tienen esta finalidad, la «acreditación» de las competencias, capacidades, saberes de una materia. Esta finalidad no puede visualizarse claramente entre los docentes que respondieron la encuesta dado que solo tres escritos mencionan la «acreditación» como propósito de un examen final.

Como ya se dijo, la clara explicitación de las finalidades de la evaluación permiten tomar decisiones ajustadas en relación al cómo evaluar, al aspecto metodológico. Es aquí donde la oralidad y la presencialidad se presentan como las opciones más valoradas, elegidas y ponderadas por los docentes. Se construyeron las siguientes categorías a partir de las respuestas obtenidas:

Metodología más adecuada para los exámenes finales:

- $1^{\circ}$ Oral, $34 \%$

- $2^{\circ}$ Presencial y oral, $28 \%$

- $3^{\circ}$ Mixta, $20 \%$ (combinación de presencialidad y virtualidad)

- $4^{\circ}$ Cuestionario virtual, $14 \%$

- $5^{\circ}$ Oral virtual, $8 \%$

- $6^{\circ}$ Presencial, $6 \%$

- $6^{\circ}$ Oral y escrito, $6 \%$

Lo enunciado aquí guarda una estrecha vinculación con las opciones metodológicas que efectivamente tomaron los docentes para el cuarto turno de exámenes, tanto los que 
cambiaron como los que sostuvieron sus metodologías de evaluación. Queda claro que, más allá de los propósitos de un examen final, en el aspecto metodológico los docentes utilizan las herramientas metodológicas que consideran como las más adecuadas, ya sea en la presencialidad o intentando recrearlas en la virtualidad.

\section{Para concluir}

En los formatos evaluativos se ponen de manifiesto las concepciones de enseñanza y de aprendizaje que subyacen en las prácticas docentes. Estas concepciones no suelen ser analizadas, revisadas y redefinidas durante la formación pedagógica de aquellos profesionales que sí las tienen, ni suelen ser revisadas en aquellos que trabajan de docentes sin esa formación. El trabajo que se pretendió impulsar desde el taller de evaluación en la carrera de Ingeniería estuvo centrado en revisar y mejorar los instrumentos y el formato de las evaluaciones que se concretaron en el cuarto turno ordinario de exámenes finales, tomando las competencias profesionales como objetivos formativos centrales.

Las acciones que efectivamente se concretaron en este sentido pusieron de relieve actitudes, creencias y prácticas en relación con la evaluación, que inician un camino de análisis de la propia práctica, un camino de investigación formativa en el equipo docente de la carrera. El análisis de coherencia-incoherencia presentado permite focalizar la mirada en los elementos que urge que sean puestos en discusión, como también la dificultad de contar con finalidades claras y pertinentes de los diferentes tipos de evaluación que se ponen en juego en el proceso formativo. Nuevamente, es necesario remarcar la importancia de ajustar las prácticas de enseñanza y de evaluación a las competencias profesionales específicas de la ingeniería.

El actual contexto de emergencia sociosanitaria, causada por la pandemia de Covid-19, obligó a todas las instituciones educativas a concretar profundos y radicales cambios para sostener los procesos formativos de los estudiantes. Los docentes, profesionales que transitaron su formación y su experiencia laboral en sistemas presenciales, debieron asumir un nuevo contexto de trabajo: el aula virtual. En mayor o menor medida se modificaron prácticas, actividades, tiempos, relaciones; pero casi en ningún caso se modificaron creencias y supuestos básicos del campo pedagógico.

Los cambios y la mejora que se concretaron en el cuarto turno de exámenes, la proactividad puesta en evidencia por el cuerpo docente, requieren continuar con acciones de formación, para que no queden solamente en pinceladas superficiales y como respuestas rápidas y eficientes, es cierto, pero no suficientes, a las exigencias de la jefatura de carrera. El incremento de la incoherencia negativa señalada anteriormente obliga a continuar profundizando las prácticas evaluativas, no solo en las instancias de exámenes finales, sino durante todo el proceso de cursado de las materias.

La mejora de las prácticas docentes continúa siendo el horizonte hacia el cual nos dirigimos: la formación por competencias de los ingenieros, pensada como un fin y, a la vez, como un medio para la mejora de la formación profesional.

\section{Referencias bibliográficas}

Adams, J. W.; Hitch, G. J. (1997). «Working Memory and Children's Mental Addition». Fournal of Experimental Child Psychology, 67, 21-38. 
CONFEDI (2018, octubre). «Propuesta de estándares de segunda generación para la acreditación de carreras de ingeniería en la República Argentina». Libro rojo de CONFEDI. Universidad FASTA Ediciones, primera edición.

Engle, R.W. (2002). «Working Memory Capacity as Executive Attention». Current Directions in Psychological Science, 11, 19-23.

Elola, N. y Toranzos, L. (2009). «Evaluación educativa: una aproximación conceptual». En Hacia una cultura de la evaluación. ONE 2009. Buenos Aires: DiNIECE. Ministerio de Educación de la Nación.

García Ramos, D. C.; Zurita Aimacaña, L. E. (2020). «Estrés académico y su relación con la memoria de trabajo en estudiantes universitarios» Revista Científica de UCES, vol. XVI n. ${ }^{\circ}$ 2. Disponible en: http://repositorio.uta.edu.ec/handle/123456789/31119 Consulta: 10 de septiembre de 2020 .

Giordano R. (2016). "Competencias y perfil del ingeniero iberoamericano, formación de profesores y desarrollo tecnológico e innovación». En Documentos Plan Estratégico Asibei. ASIBEI, 15-37.
González Agudelo, E. M. (2006). «La investigación formativa como una posibilidad para articular las funciones universitarias de la investigación, la extensión y la docencia». Revista Educación y Pedagogía, Medellín, Universidad de Antioquia, Facultad de Educación, vol. XVIII, n. 46 (septiembrediciembre), pp. 101-109.

Ministerio de Educación de la Nación Argentina (2006). Resolución n. ${ }^{\circ}$ 1456/2006. «Educación Superior Título de Ingeniero en Telecomunicaciones». Boletín Nacional 10/11/2006.

Musso, M. F.; Costa Lieste, E. y Duarte, C. (2012). «Procesos cognitivos, estrategias de aprendizaje y competencias: un estudio descriptivo en estudiantes universitarios». Revista Científica de UCES, 16(2), 127144. Disponible en: http://dspace.uces. edu.ar:8180/xmlui/handle/123456789/1908

Consulta: 10 de septiembre de 2020.

Palacios Navarro, S. (2006). «Efectos de la evaluación formativa virtual en el rendimiento académico». Revista Innovación Educativa n. ${ }^{\circ} 16$, ISSN 1130-8656, 2006, 47-57. 